Autorenfassung: Heinrich Rickerts Anpassung an den Nationalsozialismus (Zum 27. Januar 1998)

erstveröffentlicht in: Deutsche Zeitschrift für Philosophie, Band 47 (1999), S. 253-269

Hans Friedrich Fulda 1999 / 2014 
Inhalt

I. Rickert als akademischer Lehrer anno 1933/34 — Tatsachen und Zeugnisse 254

II. Zur Erklärung der ermittelten Tatbestände $\quad 258$

$\begin{array}{ll}\text { III. Ein kurzes Fazit für uns } & 268\end{array}$

\section{Heinrich Rickerts Anpassung an den Nationalsozialismus}

(Zum 27. Januar 1998)

$$
\text { von Hans Friedrich Fulda (Heidelberg) }
$$

Wer an einem Tag, an welchem sich die Befreiung des Konzentrationslagers Auschwitz jährt, mit dem Philosophischen Seminar seiner Universität der Opfer nationalsozialistischer Verbrechen gedenkt, sollte vorab beachten: Ein Philosophisches Seminar bietet der Erinnerung nicht die schützende, Gefühle in Übereinstimmung bringende Hülle einer - kirchlichen, politischen oder wenigstens ästhetischen - Zeremonie. Umso mehr hat man sich bei solchem Gedenken vor deplazierten Gesten und falschen Tönen ${ }^{1}$ in acht zu nehmen. Dicht beieinander liegen zahlreiche, sich wechselseitig ausschließende Gefahren: des unglaubwürdigen, wenn auch vielleicht virtuosen Ausdrucks von Betroffenheit oder aber eines dilettantisch hilflosen Gestammels; der Verstocktheit, weder von Mitschuld noch von Mithaftung etwas wissen zu wollen, oder der anklägerischen Überheblichkeit oder aber eines moralischen, wo nicht gar politischen Flagellantentums der Nachgeborenen, d. h. Epigonen, zu denen mittlerweile die meisten von uns gehören: Sollten wir es, um all dem zu entgehen, nicht am besten mit Gottfried Benn halten (einem freilich nicht Unbelasteten)? Der hat die sich mit ihrer Geschichte quälenden Deutschen einmal schnod- $\{\mid$ S. 254\} drig aufgefordert (ich zitiere aus dem Gedächtnis): „Haltet doch endlich die Schnauze über Eure Kaldaunen! Oder wie der Psalmist sagt: ,Meine Seele ist stille zu Gott‘.“

Wenn wir es „endlich“ dabei belassen könnten! Es dabei zu belassen aber verbietet sich aus einem zwingenden Grund. Gewiß, um aus Aischylos' Eumeniden zu zitieren: „Die Zeit, wenn sie alt wird, macht alles heil.“ Nur ist es längst nicht so weit, wie sich immer wieder zeigt. Noch haben wir es nicht mit den Eumeniden zu tun, sondern mit den Erinnyen. Über die aber belehrt uns im selben Drama Athene, die Göttin der Philosophen: ,[...] gewaltig und schwer zu versöhnen sind diese Gottheiten; über allem, was Menschen angeht, walten sie ihres Amtes. Wer ihre Ungnade auf sich lädt, den treffen die Schläge im Leben und er weiß nicht von wo. Die Vergehen der Vorfahren zwingen ihn in ihre Gewalt, und schweigend zermürbt ihn, so groß er auch prahlt, ihr feindlicher Zorn zu Staub und er geht unter.“ Das denen ins Stammbuch, die obenhin von der Gnade

\footnotetext{
${ }^{1}$ Die lauern bereits in der Rede von „Opfern des Nationalsozialismus“. Substantivische Ausdrücke empfangen ihren Sinn in der Regel von den stammverwandten Verben her. Insofern sollte man denken, es lasse sich eigentlich nur von Opfern reden, wo geopfert wird bzw. worden ist. Es müßte also, wenn jemand Opfer ist, eigentlich jemanden oder etwas geben, der bzw. das ihn geopfert hat: oder dem er - man denke an Verwendungen des Ausdrucks „Verkehrsopfer“! - sozusagen ,zum Opfer gefallen“ ist. Wenn man es mit der Sprache genau nimmt, muß man sagen: In keiner dieser beiden Bedeutungen sind diejenigen, welche durch nationalsozialistische Verbrechen zu leiden hatten oder den Tod fanden, ohne selbst $\mathrm{zu}$ den Nationalsozialisten zu gehören, „Opfer des Nationalsozialismus“ zu nennen, - es sei denn in der Perspektive Himmlers. In der haben die nationalsozialistischen Verbrecher ihrer Sache das Opfer gebracht, entsetzliche Schuld auf sich zu laden, und andere sind wie Schlachttiere diesem Opfer zugefallen, indem sie dem Nationalsozialismus „zum Opfer“ fielen. In einem solchen Sinn oder gar im Sinn der Opfer, welche die Nationalsozialisten der Durchsetzung ihrer Ziele gebracht haben, wagt heute glücklicherweise niemand, „Opfer des Nationalsozialismus“ zu sagen. Aber erlaubt uns das, diesem Ausdruck eine Bedeutung zuzuschreiben, welche unsere Sprache eigentlich nicht hergibt? Erschleichen wir uns damit nicht den Eindruck, unter allen, die irgendwie von den Verbrechen in Mitleidenschaft gezogen wurden, bestehe — uns eingeschlossen — eine Gemeinschaft mit den Opfern der Verbrechen, während die Verbrecher diese Gemeinschaft in Wahrheit gerade zerstört haben?
} 
der späten Geburt reden; aber auch jenen Jungdeutschen, die meinen, sie hätten das Recht und die Rolle, als Ankläger gegen die Älteren aufzutreten; oder es käme jetzt darauf an, daß sie feststellen, wer für was moralisch verantwortlich ist. Das Problem für uns, die 1945 noch nicht erwachsen oder nicht einmal geboren waren, ist keines moralischer Schuld anderer. Es ist das Problem kollektiv begangener Verbrechen, deren üble Folgen alle zu tragen haben - ob moralisch schuldig oder nicht, ob Nachfahren von Tätern oder von Opfern. Es ist das Problem, wie man umzugehen hat mit dem Verfolgtwerden von einer mentalen Zerrüttung, die alle immer wieder heimsucht. Wir müssen sehen, wie wir den Erinnyen unter uns Wohnung geben können, um sie uns zu Eumeniden zu stimmen

Wie soll das da gelingen, wo wir leben und arbeiten, also z. B. an einem Philosophischen Seminar? Sicher nicht so, daß wir uns wie vorm Fernseher immer wieder den Anblick gräßlichster Ereignisse verschaffen; sondern sozusagen vor Ort unseres alltäglichen Lebens — nach der Devise „Bleib“ bei Deinem Leisten; kehr vor der eigenen Tür! Besinn Dich, was da an Üblem anfing; und sei`s auch nur ein kleiner, fast verschwindend kleiner Teil des großen kollektiven Unheils gewesen!“. Daher mein Thema: Heinrich Rickert, Direktor des Philosophischen Seminars der Universität Heidelberg, anno 1933.

Was dazu im folgenden ausgeführt wird, gliedert sich in drei Teile: einen ersten, der einige Tatsachen und Zeugnisse zusammenstellt zu Rickerts Verhalten im Frühjahr 1933 und als akademischer Lehrer im Wintersemester 1933/34; einen zweiten, umfangreichsten, in dem ich mich frage, aus welchen für Rickert erkennbaren Gründen sich dieses Verhalten erklärt und als was es dementsprechend zu betrachten ist; und einen dritten, in welchem daraus ein kurzes Fazit für uns gezogen werden soll.

\section{Rickert als akademischer Lehrer anno 1933/34 — Tatsachen und Zeugnisse}

Die Tatsachen, die es hier zur Kenntnis zu nehmen gilt, sind nicht schockierend oder sensationell. Vielleicht wirken sie belanglos. Das ist mir gerade recht. So wird aus ihnen kein Anlaß, mit Fingern auf einen zu zeigen, der bisher von derartiger Aufmerksamkeit verschont war und in Sachen politischer Korrektheit eher einen guten Ruf hat. Ich muß mich in keine große Gefahr begeben, jemandes Recht auf guten Namen nach dem Tode $^{2}$ zu ver- $\{\mid$ S. 255$\}$ letzen. Ohne wie ein Ankläger zu wirken, kann ich die Tatsachen nennen, bevor ich sie in einen größeren Kontext einfüge, in dem sie uns Stoff zum Nachdenken geben.

Rickert ist im Frühjahr 193370 Jahre alt geworden. Im Wintersemester 1933/34 hat er eine Vorlesung über Fichte gehalten ${ }^{3}$, deren Typoskript die Universitätsbibliothek Heidelberg aufbewahrt. Darin hat Rickert sich einleitend auf die Ereignisse des Jahres 1933 bezogen. Er hat keinen Zweifel daran gelassen, daß er mit ihnen sympathisiert und daß die „nationale Erhebung“, wie er im Einklang mit dem Jargon der offiziellen Propaganda die Zustimmung zu Hitlers Regierungsantritt nennt, der Grund ist, aus dem er jetzt eine Vorlesung über Fichte hält. Der sachliche Zusammenhang zwischen dem Gegenstand der Vorlesung und den Ereignissen in Deutschland soll in Rickerts Augen sein: Fichte war der erste Philosoph, der in der Staatslehre sozial dachte; außerdem hat er so tief wie keiner zuvor begründet, daß die Philosophie und insbesondere die des Staats entwickelt werden muß aus dem Prinzip einer Nation, in der sie steht. Fichte hat, wie Rickert sagt, „das Nationalprinzip so tief begründet wie keiner vor ihm“. Ohne zu widersprechen, erwähnt Rickert, daß man Fichte deshalb geradezu als Nationalsozialisten bezeichnet habe. ${ }^{4}$ Er selbst, Rickert, geht nicht so weit. Aber er

\footnotetext{
${ }^{2}$ Vgl. I. Kant, Metaphysische Anfangsgründe der Rechtslehre, § 35.

${ }^{3}$ Titel: Fichtes Leben und Lehre: Zur Einführung in den deutschen Idealismus.

${ }^{4}$ Vermutlich ist dabei gedacht an: Ernst Bergmann, Fichte und der Nationalsozialismus, Breslau 1933, S. 7.
} 
bezeichnet Fichte als den frühesten und bedeutendsten Vorläufer der „Bewegung unserer Tage“; als „Vorläufer dessen, was uns heute [d. h.1933] $]^{5}$ als Nationalsozialismus umgibt“". Durch diese Vorläuferschaft besitzt Fichte für Rickert nun, 1933, aktuelles Interesse. Seine Geistesverwandtschaft mit der Weltanschauung des Nationalsozialismus soll u. a. auch darin bestehen, daß Fichte im Unterschied zu den Sozialisten des 19. Jahrhunderts nicht kosmopolitisch, nicht weltbürgerlich dachte und daß er damit kein Denken repräsentierte, wie es im Marxismus, ja, in der gesamten späteren Sozialdemokratie gipfele, - also bei den Parteien, deren Anhänger von den Nazis inzwischen verfolgt werden.

Die Einleitung zur Vorlesung, der diese Äußerungen entnommen sind, ist in einem emphatischen Stil ${ }^{6}$ gehalten, von dem Rickerts Vorlesungen sonst nichts an sich haben. Kennzeichen dieses Stils sind leidenschaftliche Beurteilungen — positiv wie negativ — von zeitgeschichtlichen und älteren Tatbeständen. Zum Beispiel wird der Versailler Vertrag als etwas „Ungeheuerliches“ charakterisiert; als ein „monströses Haßprodukt“, wie es „,die Weltgeschichte noch nicht gesehen hat““. Der Völkerbund wird als „Theater“ abgetan. Im Kontrast zu diesen europapolitischen Gegebenheiten wird Fichte heroisiert. Er habe „sich der Weltmacht [Napoleon] als einzelner entgegengestellt“. In seinem Charakter sei „nichts Halbes“, sondern „Ganzheit und Kraft“. Er sei „,von antiker Größe“. — Soweit die wichtigsten Äußerungen Rickerts, über die nachzudenken ich hier anregen will. Wer sich unter deutschen Professoren-Verlautbarungen des Jahres 1933 umgetan hat, wird wissen: die Äußerungen sind nichts, was aus dem Rahmen fiel. Im Vergleich zu Anderem, was damals \{|S. 256\} gesagt wurde, sind sie sogar ziemlich harmlos — von den Verbrechen, die begangen wurden, ganz zu schweigen. Anstoß zum Nachdenklich-werden geht davon nur aus, wenn man die Äußerungen in den Kontext zurückstellt, dem sie entstammen; in den Kontext desjenigen nämlich, der Heinrich Rickert war, und der Philosophie, für die er stand. Darum nun hierzu einige Bemerkungen und in ihnen Hinweise auf weitere Zeugnisse.

Nach seiner politischen Einstellung gehörte Rickert in den Jahren der Weimarer Republik nicht zu den Nationalkonservativen, die an der Universität die zahlreichste Gruppe bildeten, sondern — wenn auch nicht ganz eindeutig - zur liberalen Heidelberger Professorengruppe um Max und Marianne Weber, mit deren Kreis er auch nach dem Tod von Max Weber verbunden blieb. Er war den Zielen der Kathedersozialisten und dem christlichen Sozialismus Friedrich Naumanns gegenüber aufgeschlossen, selbst aber nach seiner Herkunft ungefähr auf der Linie der DDP. In den früheren Jahren der Weimarer Republik und vor dem Zerfall des liberalen Lagers dürfte er diese Partei gewählt haben.

Rickerts Mitgehen mit der angeblichen „nationalen Erhebung“ signalisiert nicht plötzliche politische Erregtheit eines bis dahin Unpolitischen, der auf einmal „politisiert“ ist, aber immer noch keine Ahnung von dem hat, was vor sich geht. Das trifft auf den Rickert-Schüler und -Intimus Hermann Glockner zu, nicht aber auf Rickert selbst. Der war ein Mann von politischer Bildung. Er hatte mannigfache persönliche Beziehungen zu Politikern und schenkte öffentlichen Vorgängen dauerhaft Aufmerksamkeit. Dies übrigens schon von seinem Danziger und später Berliner Elternhaus her: Sein Vater war in Danzig Journalist gewesen, war Mitbegründer der Freisinnigen Partei und Reichstagsabgeordneter. Die führenden Politiker der „Gründerzeit“ waren im Haus des Vaters aus- und eingegangen; und der Sohn hatte sich schon als Gymnasiast (am renommierten Berliner

\footnotetext{
${ }^{5}$ Eckige Klammern und ihr Inhalt sind mein Zusatz.

${ }^{6}$ Auf den hat mich Robert Schnepf (Halle) aufmerksam gemacht.

${ }^{7}$ Als hätte die Welt nicht kurz vor Versailles den in Brest-Litowsk von den Deutschen diktierten Friedensvertrag gesehen, nach welchem Rußland ein Viertel seiner Bevölkerung und Landfläche sowie drei Viertel der Eisen- und Kohleproduktion verlor!
} 
„Grauen Kloster“) journalistisch betätigt. Trotz solcher Fähigkeiten waren übrigens seine Vorlesungen einschläfernd nüchtern und langweilig. Nun aber spricht Rickert auf einmal mit Pathos.

Rickerts Abkehr von der Weimarer Republik kündigt sich nicht bereits zu Beginn der dreißiger Jahre an, d. h. während der Weltwirtschaftskrise und der Notverordnungsregierungen; aber nicht in politischen Äußerungen zum Tagesgeschehen — im Unterschied zur Entwicklung bei Willy Hellpach, einem ausgeprägteren Repräsentanten der liberalen Heidelberger Professorenschaft. Man erkennt Rickert aus den uns von ihm selbst erhaltenen Zeugnissen daher nicht als einen von denen, die mit Beginn der dreißiger Jahre an der parlamentarischen Demokratie irre wurden und sich auf den „Mittelweg nach rechts“ ${ }^{\text {“8 }}$ begaben.

Rickerts Bekenntnis zur „nationalen Erhebung“, welche die Nationalsozialisten inszenieren, ist auch nicht einfach einer momentanen Suggestion im politischen Frühjahrstaumel des Jahres 1933 geschuldet. Das Bekenntnis hält jedenfalls das Jahr über und ins nächste Jahr hinein an — trotz der Verfolgungen, die gleich nach der Machtübernahme einsetzten, trotz der Verletzung von elementaren Menschenrechten nicht zuletzt an der Universität, trotz der Verbrechen, die das Regime begeht. Allerdings unterscheidet sich Rickert da- $\{\mid S$. $257\}$ durch nicht von vielen anderen derjenigen ,,bürgerlichen“ Hochschullehrer, für welche die Politik der Nazis keine unmittelbare Bedrohung im privaten und beruflichen Alltag bedeutete.

Man könnte denken: Was Rickert in seiner Vorlesung sagt und an Engagement zu erkennen gibt, das soll nur zur Tarnung dienen und zum Schutz persönlicher Interessen. Angesichts des erheblichen Drucks, der von nazistischen Hörern ausgegangen sein dürfte, wäre es ja auch eine verzeihliche Unredlichkeit gewesen, vielleicht sogar ein passabler hochschuldidaktischer Trick. Aber es handelt sich bei den Äußerungen nicht um ein aus der Not geborenes Lippenbekenntnis. Rickert ist über siebzigjährig und gar nicht mehr verpflichtet, eine Vorlesung zu halten. Er legt Wert darauf, nicht aus Opportunismus nun über Fichte zu lesen und von ihm zu sagen, was er sagt: In seiner Vorlesung dürfe man „keine Akkommodation an die gegenwärtige Lage erblicken“. Er spreche „,aus Überzeugungen, die sein ganzes Leben durchziehen“. Dazu muß man wissen, daß die Nazis inzwischen denen, die nun, nach der „Machtergreifung“, in ihre Nähe drängen, die kalte Schulter zeigen. Rickert grenzt sich mit seinen Äußerungen also von den „Märzgefallenen“ ab und spricht sich das Recht zu, sich zur „Bewegung“, soweit sie in Fichte nach Rickerts Meinung ihren Vorläufer hat, zu bekennen. Daß darin bei Rickert inzwischen auch eine Zustimmung zu Hitlers Machtanspruch enthalten ist, bezeugt Hermann Glockner. Er führte in seiner ganzen Heidelberger Zeit regelmäßig Tagebuch. Auf der Basis derart befestigter Erinnerung schrieb er im Sommer 1945 (,,in trüben Stunden“) einen persönlichen Lebenslauf. Darin heißt es in Bezug auf die Spätphase der Weimarer Republik über ihn selbst und Rickert: „An der Parteipolitik der damaligen Jahre nahm ich nach wie vor keinen Anteil, obwohl im Hause Rickert [wo Glockner wohnte] [...] viel politisiert wurde. [...] Gab es eine Wahl, so ließ ich mich von Rickert beraten. Wir wählten dann demokratisch oder Deutsche Volkspartei, obwohl meine Frau sozialdemokratische Jugendneigungen schwer unterdrücken konnte. [...] Hitler wurde von Rickert in jener Zeit noch völlig abgelehnt. Erst 1933 ließ er sich durch Hitlers Erfolge umstimmen. Er suggerierte sich damals, daß im Grunde Fichte der erste Nationalsozialist gewesen sei, denn Fichte wäre sowohl Nationalist wie Sozialist gewesen. “9

\footnotetext{
${ }^{8}$ Vgl. dazu die Heidelberger Dissertation von Christian Jansen, Auf dem Mittelweg nach rechts. Politisches Denken und Handeln von Hochschullehrern an einer liberalen Universität: Heidelberg 1914-35 (1989). Darin ist charakteristischerweise nicht registriert, was mit Rickert vor sich ging.

${ }^{9}$ Glockner-Archiv in Fürth.
} 
$\mathrm{Da}$ es so war, wird auch durch Zeugnisse über den Rickert-Assistenten August Faust bestätigt. Der ließ sich von der Suggestion Rickerts, was Fichte und den Nationalsozialismus betrifft, anstecken und konvertierte im Frühjahr 1933 buchstäblich über Nacht zum Nationalsozialisten ${ }^{10}$, der er auch bis zu seinem Selbstmord im Jahr 1945 blieb. - Soweit die Entwicklung der Rickertschen Präferenz für Parteien. Man darf bei dieser Entwicklung im Hinblick auf Rickerts Philosophie jedoch nicht vergessen, daß es sich um Weltanschauungsparteien handelte und daß Rickert in Sachen politischer Weltanschauung alles andere als ahnungslos war. Er war hierin der Experte. Denn zeitlebens hat er die Philosophie als Weltanschauungslehre verstanden.

Hinsichtlich Rickerts Parteinahme für den Nationalsozialismus, wie sie in der Vorlesung über Fichte zum Ausdruck kommt, zeichnet sich damit ab: wenn man sie erklären will, so bietet sich keines der Muster an, die andernorts gefunden und beschrieben worden sind. \{|S. 258\}

Rickerts Parteinahme oder zumindest ausdrücklich gemachte Affinität zum Nationalsozialismus ist keine kleine, nebensächliche Konzession an erregte oder gar drohende Hörer-Erwartungen; sie bringt nicht zum Ausdruck, daß ein bislang Unpolitischer plötzlich zu Mitläuferschaft stimuliert ist und das womöglich aus Privatinteressen, wie z. B. denen einer Karriere; Rickerts Parteinahme für die Nazis folgt auch nicht dem üblichen Muster verzweifelter Versuche, sich tagespolitisch neu zu orientieren, wie man das von anderen zuvor liberal Gewesenen kennt. Denn sie erfolgt in Besinnung auf eine weltanschauliche Grundüberzeugung, die längst besteht. Aber die Parteinahme kommt auch nicht dadurch zustande, daß nun auf einmal bisher verdrängte Regungen oder unterdrückte anarchische Motive durchschlagen, wie es bei manchem Antisemiten der Fall war. Nach dem Zeugnis Glockners galt Rickert als Gegner des Antisemitismus. Nichts, soweit ich sehe, spricht dafür, daß er auf einmal in seinem Innersten (oder aus ihm heraus) aufhörte, es zu sein, und das Gegenteil davon wurde. - Wie aber verhielt es sich dann? Um das auszumachen, müssen wir nach erklärenden Gründen suchen. Bisher aber haben wir nur Hinweise auf Richtungen, denen folgend man eine Erklärung für Rickerts Äußerungen und politische Einstellung anno 1933 nicht suchen darf. Was erklärt uns Rickerts Einstellungsänderung positiv — soweit wenigstens, daß wir sie aus Gründen verstehen können, mögen wir auch weit entfernt sein, die Gründe zu teilen und ihre Folgen zu billigen?

\section{Zur Erkklärung der ermittelten Tatbestände}

Ich sehe dafür zwei Ansätze. Sie schließen einander nicht aus. Der eine Erklärungsansatz ist leichter zugänglich als der andere, trägt aber alleine nicht weit genug — und dies nicht nur im Hinblick auf die Erklärungsleistung, sondern vor allem im Hinblick auf den Inhalt der Rickertschen Philosophie. Für den nämlich müssen wir uns letztlich interessieren, wenn wir nach einer in sich stimmigen Erklärung suchen, die zugleich erlaubt, die Gründe für Rickerts Äußerungen und Einstellung zum Nationalsozialismus zu beurteilen. Ich kann auch nur mit diesem Interesse nach der eingangs ausgegebenen Devise verfahren, „bei meinem Leisten“ zu bleiben.

A) Der für den ersten Erklärungsansatz zu beachtende Kontext ist die lebensgeschichtliche Situation, in der sich Rickert anno 1933 befindet — mit einer zunächst sehr düsteren Einschätzung der Zukunft für sein philosophisches Werk. Hierzu noch ein paar biographische Einzelheiten. Kurz bevor Rickert seinen 70. Geburtstag begeht (25.5.1933), steht in der Fakultät die Frage nach seiner Nachfolge an. Im Januar sieht es so aus, als könne sein Lehrstuhl aus Einsparungszwängen nicht wieder besetzt werden — jedenfalls nicht mit einem

${ }^{10}$ Vgl. dazu den Bericht R. Klibanskys in: Unispiegel, 6/88. Der Held der darin erzählten Geschichte läßt sich mithilfe von Glockners Heidelberger Bilderbuch (Bonn 1969) leicht identifizieren. 
Philosophen. Über die Wirkung, die das auf Rickert hatte, notiert sich Glockner in sein Tagebuch (15. Jan. 33) ${ }^{11}$ : „wieder einmal ein sehr deprimierender Besuch bei Rickert (der weil er selbst am Ende ist, alles am Ende sieht und sich sogar in der Betrachtung des Zusammenbruchs der Philosophie in Heidelberg nach seinem Abgang gefällt!) und die noch niederdrückendere letzte Fakultätssitzung, in der neue Sparmaßnahmen besprochen wurden und sozusagen beschlossen wurde, daß Rickert keinen Nachfolger erhält.“ $\{\mid$ S. 259\}

Wenn wir uns in die Lebenslage Rickerts versetzen und Glockners Heidelberger Bilderbuch trotz seiner literarischen Überformung biographischer Tatbestände ${ }^{12}$ Glauben schenken dürfen, werden wir noch etwas anderes registrieren: Seit einigen Jahren macht Heidegger von sich reden, den Rickert (noch in Freiburg) einmal für kurze Zeit als seinen Schüler betrachten konnte; Jaspers, der Kollege am Ort, steht mit Rickert auf sehr gespanntem Fuß, zieht aber viele der Jüngeren an, z. B. auch den letzten Rickert-Schüler und -Assistenten Franz Böhm. Heidegger und Jaspers treten als Kombattanten für eine neue, mit existenziellem Ernst betriebene Philosophie auf und werden auch als solche wahrgenommen. Sie aber geben deutlich zu verstehen, wie gering sie vom Wert des Rickertschen philosophischen Werks denken. Glockner registrierte, wie sehr dies Rickert zu schaffen machte, wie auch die Erfolge der phänomenologischen Schule ${ }^{13}$, der sich Heidegger nach Rickerts Weggang aus Freiburg angeschlossen hatte. Rickerts Reaktion auf diese bedrückenden Umstände — schon vor 1933 - deutet Glockner so: „Er wollte zeigen, daß er keineswegs gegen den Strom schwimme, sondern mit der Jugend jung geblieben sei“. ${ }^{14}$

Könnte es da nicht sein, daß Rickert sich aus der niederdrückenden Stimmung des Januar 1933 nun mit einem letzten Kraftakt zu befreien sucht, indem er seine Philosophie den Nationalsozialisten andient - als ihrer „Weltanschauung“ im Grunde adäquat - zumindest partiell, nämlich was die Verbindung des nationalen und des sozialistischen Ingrediens dieser sogenannten Weltanschauung betrifft? Indem Rickert selbst in seiner Sozialphilosophie diese Verbindung seit vielen Jahren empfiehlt und indem er sich dafür auf Fichte beruft, den Erwecker der deutschen Nation zum Kampf gegen die französisch-napoleonische Vor- und Fremdherrschaft, kann er sich doch ebensogut als einen Nationalsozialisten avant la lettre betrachten oder zumindest als einen natürlichen philosophischen Verbündeten der Nazis. Mit Genugtuung scheint er registriert zu haben, daß Jaspers dies nicht konnte und nun in eine akademische Randstellung gedrängt wurde. ${ }^{15}$

Solche Motive, deren Bild man noch verfeinern könnte ${ }^{16}$, mögen eine Rolle gespielt haben. Aber wenn man nur sie und keine anderen unterstellt, bleiben zwei Gesichtspunkte unberücksichtigt, die nicht erst aus unserer Sicht relevant sind, sondern auch in Rickerts Augen Beachtung verdienen mußten, während sie die Dominanz oder gar Alleinherrschaft so persönlicher Motive geradezu monströs erscheinen lassen:

1. Ähnlich wie Max Weber die Forderung erhob, sozialwissenschaftliche Forschung und Lehre wertfrei zu betreiben, vertrat Rickert selbst in Zeiten größter politischer Erregung stets den Grundsatz, daß die Hochschullehrer nicht vom Katheder herab zum politischen Tagesgeschehen Stellung nehmen sollen. Auch in seiner Fichte-Vorlesung von 1933/34 macht er auf diese Maxime aufmerksam. Unmittelbar bevor er sich

\footnotetext{
${ }^{11}$ Glockner-Archiv in Fürth.

12 Glockners Liebe gilt der Belletristik. Um seinem Bilderbuch gerecht zu werden, darf man es nicht mit Wissenschaftler- oder Politiker-Memoiren gleichsetzen. Man muß es wie das autobiographische Erinnerungsbuch eines Prosaschriftstellers lesen.

${ }^{13}$ Glockner, Heidelberger Bilderbuch, a. a. O., 98.

${ }^{14}$ Ebd., 93.

${ }^{15}$ Ebd., 98.

${ }^{16}$ Man müßte dazu auf Rickerts 70. Geburtstag und die Berufung seines Nachfolgers Ernst Krieck eingehen.
} 
dagegen verwahrt, daß $\{\mid$ S. 260\} man ihm sein Eingehen auf Fichtes nationalen Sozialismus als Akkommodation an die momentane Lage deute, sagt er: „mir liegt Tagespolitik auf dem Katheder als Hauptsache sehr fern“. Wenn er trotzdem das große aktuelle Interesse betont, das Fichte als nationaler Denker gerade jetzt verdient, muß er der Auffassung sein, daß die von den Nationalsozialisten inszenierte „,nationale Erhebung“ einen epochalen Einschnitt markiert; und daß dadurch seine Stellungnahme zum Nationalsozialismus weit über die Kommentierung von Tagespolitik hinausgehoben wird. Darauf deuten auch die starken Worte zum Versailler Vertrag hin als einem Haßprodukt, wie es die Weltgeschichte noch nicht gesehen hat, und zu Fichte als einem Mann von antiker Größe. Was aber sollen in solchem Kontext die erwähnten persönlichen Motive selbst wenn man Rickert ein gewisses Maß an professoraler Eitelkeit zusprechen muß?

2. Wichtiger ist ein anderer Gesichtspunkt, unter dem der bisherige Erklärungsansatz problematisiert oder wenigstens relativiert werden sollte. In Deutschland finden 1933 seit Hitlers „Machtergreifung“ von der Regierung ausgehende Verfolgungen statt, mag auch das Ausmaß dessen, worauf es dabei abgesehen ist, selbst für einen politisch Gebildeten wie Rickert nicht zu überschauen sein. Menschenrechte, Prinzipien des Rechtsstaats werden mit Füßen getreten. Deutschland verläßt den Völkerbund. Die parlamentarische Demokratie und alle Möglichkeiten zu legaler Opposition werden beseitigt, die machthabende Partei und ihre Führung definieren, wer zur Volksgemeinschaft gehört und wer aus ihr ausgegrenzt, ins soziale Nichts gestoßen, vertrieben oder im KZ drangsaliert werden soll. All dies geschieht unter Berufung auf eine nationalsozialistische „Weltanschauung“. Das ist es, was Rickert und seine Hörer im November 1933 ,als Nationalsozialismus umgibt“" Wer wie Rickert Philosophie als Weltanschauungslehre betreibt, kann nicht daran vorbeisehen. Er muß sich fragen, was das alles bedeutet, wie er darüber im Licht seiner Lehre zu urteilen hat und wie gut oder schlecht dieses Geschehen zur eigenen Weltanschauung paßt, die doch durch wissenschaftliche Weltanschauungslehre aufgeklärt sein soll. Wenn wir nach einem erklärenden Motiv für Rickerts Äußerungen Ausschau halten, werden wir uns also unter den Inhalten der Rickertschen Weltanschauungslehre umsehen müssen.

B) Damit bin ich beim zweiten Erklärungsansatz und mit ihm endlich beim philosophischen Teil meiner Ausführungen. Bevor ich die Elemente zusammenstelle, aus denen dieser Erklärungsansatz besteht (2.), und dann ihn selbst skizziere (3.), sollte ich aber wohl in wenigen Worten umreißen, was für ein Verständnis von Philosophie als Weltanschauungslehre Rickert gehabt hat (1.).

1. Es war eine von Rickerts Grundüberzeugungen, daß die Wissenschaften ihre Suche nach theoretischer Erkenntnis im Rahmen von weltanschaulichen Orientierungen betreiben. Diese Orientierungen und die Weltanschauungen, von denen aus wir sie erlangen wollen, können nicht ihrerseits den Anspruch erheben, wissenschaftliche Erkenntnis $\mathrm{zu}$ sein. Wir bezeichnen eine Weltanschauung typischerweise durch einen Ausdruck, in welchem ein Adjektiv, das etwas sehr Generelles bezeichnet, durch das Suffix „,-ismus" ergänzt ist (z. B. Positivismus, Naturalismus, Materialismus, Idealismus, Vitalismus). Wenn man Weltanschauungen so versteht, ist klar, daß sie zwar ohne berechtigten Erkenntnisanspruch sind, daß aber das Bedürfnis nach ihnen den Wissenschaftlern genau so wenig auszutreiben ist wie dem Leben, das wir außerhalb der Wissenschaften führen, und den Diskussionen über Lebensangelegenheiten, in die wir uns verwickeln, — insbesondere wenn es um $\{\mid$ S. 261\} Existenzfragen geht und in solchen Fragen um gesellschaftliches, durch Recht normiertes und staatlich organisiertes Zusammenleben. Klar ist auch, daß sich unsere Weltanschauungen sowohl im Kontext theoretischer Erkenntnis als auch im Kontext der Lebenspraxis nicht friedlich wie Bilder einer Ausstellung miteinander vertragen. Sie konkurrieren - sowohl untereinander als auch um ihre orientierende Funktion, 
schließen sich aber in dieser Funktion meistens gegenseitig aus. Obwohl wir uns nicht aufgrund von theoretischer Erkenntnis allein für die eine oder andere von ihnen entscheiden können, gehört die Suche nach einer bestmöglichen Entscheidung (individuell sowie kollektiv) zu dem uns aufgetragenen „Kampf um einen geistigen Lebensinhalt“ ${ }^{17}$

Die Irrationalität dieses Kampfes und ihre unerwünschten Folgen zu minimieren ist die Aufgabe der Philosophie. Um diese Aufgabe zu erfüllen, hat die Philosophie einerseits - als Wissenschaftslehre - zu zeigen, wie die verschiedenen Wissenschaften ungeachtet verschiedener in ihnen wirksamer Weltanschauungen zu theoretischen Erkenntnissen gelangen können; andererseits hat die Philosophie aber auch die „Werte“ zu sortieren, denen man in den verschiedenen Weltanschauungen Tribut zollt. Sie hat zu untersuchen, welche von diesen Werten in welcher Bedeutsamkeit für uns mit welchen anderen Werten unverträglich sind - und welche umgekehrt mit welchen anderen so verträglich, daß sie sich zu einer wenigstens intern stimmigen „Wertsynthese“ und schließlich zu einer ganzen, kohärenten Weltanschauung zusammenfügen. In Wahrnehmung dieser Aufgabe ist die Philosophie Wertphilosophie und als solche am Ende Weltanschauungslehre. Beide Teilaufgaben - die der Wissenschaftslehre und die der Wertphilosophie bzw. Weltanschaungslehre - sind wissenschaftlich zu erfüllen. Ihre Bearbeitung steht damit unter der Forderung kontinuierlichen Ausschlusses von Irrationalität. Im Unterschied zu Max Weber hält Rickert diese Forderung auch in einer Wertphilosophie für erfüllbar. Als negatives Pendant gehört zu diesem positiven Auftrag einer wissenschaftlichen Philosophie daher die Kritik an irrationalistischen Tendenzen in der Philosophie, sofern sie einem nicht in sich stimmigen weltanschaulichen Muster huldigen. Ein Beispiel hierfür ist Rickerts Kritik an der Lebensphilosophie.

Aber von der wissenschaftlichen philosophischen Wertlehre (und der Weltanschauungslehre innerhalb ihrer) muß eine Weltanschauung selbst unterschieden werden. Sie ist nicht allein durch wissenschaftliche Arbeit zu gewinnen - und sei sie auch eine philosophisch aufgeklärte Weltanschauung. Eine solche philosophisch aufgeklärte Weltanschauung hat Rickert letztlich mit seiner Gelehrtenarbeit gesucht und in einigen Schriften wie auch in Lehrveranstaltungen - zum Ausdruck bringen wollen. Er war sich klar darüber, daß die wissenschaftliche Weltanschauungslehre dafür nur eine Vorbereitung sein kann. Sie leistet Vorarbeit nach seiner Meinung insbesondere dadurch, daß sie als wissenschaftliche Wertanalyse diejenigen Werte auszeichnet, die in sich schon, aber auch für die Zusammenhänge unseres Lebens mit seinen Kulturzielen besonders integrativ sind, so daß sie sich für Wertsynthesen eignen und sich zu einer nicht einseitigen, sondern möglichst umfassenden Weltanschauung qualifizieren. Solcher Qualifikation wegen verdienen diese Werte in einer wissenschaftlich aufgeklärten Weltanschauung vor anderen Werten Präferenz. Zur Beantwortung unserer Frage nach einem innerphilosophischen Motiv für Rickerts Äußerungen und Einstellung \{| S. 262\} zum Nationalsozialismus muß man sich also überlegen, welche der von Rickert thematisierten Werte es sind, die solche Eigenschaften besitzen, und aufgrund wovon sie eine besondere Präferenz verdienen. Damit komme ich zu den Elementen der gesuchten Erklärung.

2. Im Rickertschen Tableau möglicher Klassen von Werten, die an irgendwelchen Gütern haften, sofern diese Güter von jemandem geschätzt werden, müssen wir für unseren Zweck diejenigen Werte ins Auge fassen, deren Verwirklichung ein aktives Eintreten für sie (und nicht nur ein kontemplatives Verhalten zu ihnen) verlangt - und zwar insofern die Werte Personen zuzuordnen sind bzw. ihrem sozialen Zusammenhang (im

${ }^{17}$ Vgl. die so betitelte Schrift Rudolf Euckens von 1896. 
Unterschied beispielsweise zum Wert der Wahrheit oder den Werten ästhetischer Beurteilung). Unter den zu beachtenden Wertklassen gibt es nun nach Rickerts Auffassung aufgrund der Natur von Bewertungsakten mindestens zwei Stufen: Zum ersten diejenige im weitesten Sinne sozial-ethischer Werte, die unter entsprechenden Forderungen zu verwirklichen sind durch ein ins Unendliche gehendes, auf die Zukunft einwirkendes Streben. Die Werte dieser Stufe sind mit anderen Worten solche, deren Verwirklichung unter der dauernden Spannung einer Differenz von Sein und Sollen steht. Rickert nennt die Güter, an denen Werte dieser Stufe haften, auch Zukunftsgüter. Ihre Werte können universalistisch und kosmopolitisch konzipiert sein. Zum zweiten gibt es praktische Werte auf einer anderen Stufe, die so nicht konzipiert sein können. Für ihre Verwirklichung besteht keine Spannung zwischen Sein und Sollen. Rickert nennt sie Werte, die an Gütern des persönlichen Gegenwartslebens haften. Zu ihnen gehören Werte, die im intimen zwischenmenschlichen Bereich Geltung haben z. B. derjenige der Mutterliebe ${ }^{18}$. Es gehört dazu aber auch der Wert der Nation. Eine Nation wird dabei als ein Kulturgut verstanden, das für die ihr Angehörigen Verpflichtungen mit sich bringt. Die Nation, zu welcher jemand gehört, besitzt für den betreffenden aber nicht irgendeinen Wert dieser Stufe, sondern denjenigen des umfassendsten Gutes, das es nach Rickerts Auffassung auf dieser Wertstufe gibt. Umfassendere wertbehaftete Güter, wie z. B. die Menschheit oder das Recht, finden sich nur auf der erstgenannten Stufe und auf einer dritten, deren Güter nach Rickert jedoch ausschließlich religiöse sind. Nun werden zwar die Werte der ersten beiden Stufen als gleichrangig eingeführt. Aber von der dritten Wertstufe her, in der sich nur noch Güter religiösen Werts befinden, erhalten doch die Werte der zweiten Stufe, also auch derjenige der Nation eine, wie Rickert sagt, „gewisse Weihe“19. Durch diese „Weihe“ werden sie mit Rücksicht auf die Tendenz zu vollendeter Wertverwirklichung über die Werte der Zukunftsgüter gestellt.

Von hier aus liegt es nahe, universalistische Forderungen, die mit der Idee des Rechts und der Menschheit verbunden sind, den Verpflichtungen unterzuordnen, die wir aufgrund der Zugehörigkeit zu einer bestimmten Nation haben. Folgt man dieser Suggestion, so müßten also universalistische Rechtsforderungen zurückstehen, wenn sie sich mit Geboten der Liebe zur eigenen Nation nicht vertragen. Diese Präferenz scheint sich Rickert in seiner eigenen Weltanschauung, von seiner Wert- und Weltanschauungslehre angeregt, tatsächlich gebildet zu haben. Beleg dafür ist seine Interpretation des Goetheschen Faust. ${ }^{20}\{\mid$ S. 263\}

Denn Rickert glaubte seine Weltanschauung in diesem Werk finden und anhand von dessen Interpretation oder vielmehr an den Tätigkeiten und Einstellungen zur Welt, die dessen Held durchläuft, zum Ausdruck bringen zu können. Nach dem auf Zukunftsgüter gerichteten erotischen Eudämonismus der Gretchen-Tragödie, dem Genuß der Kunst als Gegenwartsgut in der Helena-Tragödie und der auf unendliche Vervollkommnung gerichteten Wertverwirklichung einer ethisch begründeten Weltanschauung in der Herrscher-Tragödie kommt Faust (und mit ihm der faustische deutsche Mensch) Rickerts Deutung zufolge darin zur Erfüllung seines immer strebenden irdischen Bemühens, daß er am Ende kein höheres Ziel mehr kennt, ,als innerhalb des Volkes, in dem er wirkt, für alle tätig zu sein“²1 und das tätige Leben der anderen Glieder seiner Gemeinschaft zu fördern. Rickert bemerkt nicht, was für ein finsterer „Patron“ (Goethe, Faust, Vers 11172) Faust inzwischen geworden ist, wie überheblich er glaubt, er müsse „ermüden [...] gerecht zu sein“(Vers 11272), wie verblendet er zu Werke geht und welche Schuld er mit dem Befehl, Philemon und Baucis zu beseitigen, auf sich lädt. Dem seine

\footnotetext{
18 Wenn eine Mutter von Mutterliebe erfüllt ist, dann ist sie der Grund dafür, daß sie tut, was ihr eine solche Liebe gebietet. Sie soll ein solcher nicht nur sein und soll nicht nur das betreffende tun.

${ }^{19}$ Vgl. H. Rickert, Vom System der Werte, in: Logos, Bd. IV, 1913, 304.

${ }^{20}$ H. Rickert, Goethes Faust. Die dramatische Einheit der Dichtung, 1932.

${ }^{21}$ Ebd., 535.
} 
Wertlehre in Goethes Dichtung hineinlesenden Philosophen entgeht auch, wie wenig die Lemuren, die Faust ohne sein Wissen auf Mephistopheles“ Geheiß das Grab schaufeln, den Erblindeten ,,auf freiem Grund mit freiem Volke stehn“ (Vers 11580) lassen. Das schließlich wirkungslos gewordene, doch immer noch herrische Gebaren des an seinem Ende angelangten Faust widerspricht kraß dem Traum, ,in dem immer weiter wachsenden und sich fortentwickelnden Ganzen eines tatkräftigen Volkes“ tätig zu sein „und so in dem Allgemeinen für immer Spuren seines individuellen Wirkens ${ }^{\text {‘22 }} \mathrm{zu}$ hinterlassen. Da wird keiner des angeblich umfassendsten unter den Gütern persönlichen Gegenwartslebens teilhaftig. Vielmehr wird uns das Bild einer sich in vanitas auflösenden totalitären Herrschaft gezeichnet; aber den Weltanschauungsphilosophen Rickert erreicht davon nicht der leiseste Schauder. Nicht anders ist es charakteristischerweise linken Interpreten ergangen, wie z. B. G. Lukács, der Rickert hier gedankenlos folgt. ${ }^{23}$

Der philosophische Grund, Vaterlandsliebe vor universalistischen Forderungen zu privilegieren, war für Rickert die affektive, erotische Komponente des Werts, den das Gut ,Nation“ in seinen Augen besitzt. Denn sie macht nach Rickerts Meinung den Wert der Nation bereits in sich zu einer gewissen Synthese aller Werte der ersten beiden Stufen. ${ }^{24}$ Das ist der wichtigste und fatalste Punkt. Hinzu kommt, daß die Philosophie nach Rickerts Meinung dort ihren Anspruch auf universale Verständigung aufgeben muß und nur noch nationalen Charakter haben, also primär nur noch nationale Bedeutsamkeit anstreben darf, wo sie von der wissenschaftlichen Weltanschauungslehre zu einer durch sie aufgeklärten, von Rickert überwissenschaftlich genannten philosophischen Weltanschauung übergeht. Ihre Lebensbedeutsamkeit, die sie zu suchen hat und bei deren Suche Rickert nun nicht hinter den Existenzphilosophen zurückstehen will, soll vorran- \{| S. 264\} gig (wenn nicht ausschließlich) eine nationale sein. Das ist das zweite. $\mathrm{Zu}$ ihm kommt als drittes eine bestimmte Einschätzung der kulturellen Lage, in der sich die Deutschen seit dem Ausgang des Ersten Weltkriegs befinden, und eine daraus abgeleitete Forderung an diejenigen, die Kultur schaffen. In seinem letzten Buch, das der Heidelberger Theologischen Fakultät als Dank für einen Ehrendoktor gewidmet ist, hat Rickert diese Einschätzung und Forderung am Pointiertesten ausgedrückt: ${ }^{25}$

Was wir Deutsche heute und morgen zu wollen und zu tun haben, und was insofern den ,Sinn“ unseres gegenwärtigen Lebens ausmacht, hängt von ganz anderen als philosophischen Überlegungen ab. Die Lage, in der wir momentan wirken, ist nämlich dadurch gekennzeichnet, daß die gesamte deutsche Kultur noch immer in Gefahr schwebt. Daraus wird verständlich, daß die politischen Fragen, die sich um diese Gefahr drehen, im Vordergrund stehen, denn von der Politik, die wir machen, hängt es vor allem ab, ob wir als deutsche Kulturmenschen überhaupt ,am Leben bleiben', oder ob die gesamte deutsche Kultur zugrunde geht. Deshalb sollte kein Deutscher, der in unseren Tagen innerhalb Deutschlands Kultur wirken will, sich gegen das Vorwiegen der national-politischen Kulturziele auflehnen. Falls seine außerwissenschaftliche Weltanschauung mit dem, was ,Forderung des Tages' ist, nicht übereinstimmt, sondern ihren Schwerpunkt in anderen Kulturgütern als im nationalen Staate sieht, hat er seine Ansichten über den Sinn des gegenwärtigen Lebens der historischen Situation anzupassen. Sonst muß er überhaupt auf jede Wirksamkeit im Kulturleben verzichten, da eine solche auf die Dauer nur innerhalb seines Volkes und seines Staates möglich ist.

Man denke nicht, das sei ein Beispiel für ,persecution and the art of writing“ gewesen! Es war ein Bekenntnis, abgelegt vor der höchsten Autorität einer Fakultät für Theologie.

\footnotetext{
${ }^{22}$ Ebd., 520.

${ }^{23}$ Zur Kritik an dieser Interpretation vgl.: Albrecht Schöne, Johann Wolfgang Goethe. Faust. Kommentare, in: J. W. Goethe, Sämtliche Werke. Briefe, Tagebücher und Gespräche, hg. v. F. Apel/ H. Birus u. a., I. Abteilung: Sämtliche Werke, Band 7/2, Frankfurt/M. 1994, 718 ff., bes. 747-751.

${ }^{24}$ Rickert, Vom System der Werte, a. a. O., 319.

${ }^{25}$ Rickert, Grundprobleme der Philosophie. Methodologie. Ontologie. Anthropologie, Tübingen 1934, 223.
} 
Schließlich, und damit kommt der nationale Sozialismus ins Spiel: Rickerts Sozialethik lehrt (ebenfalls längst vor 1933), der Staat, der natürlich ein Nationalstaat ist, habe nicht nur irgendwie die Kulturziele der Nation zu verfolgen. Er sei, wie schon Treitschke meinte, wesentlich eine Veranstaltung zur Organisation von Macht $^{26}$; und als solche habe er jedem die Möglichkeit zu beruflicher, Lebensunterhalt sichernder Arbeit zu schaffen; er habe daher den wirtschaftlichen Individualismus und Liberalismus zu bekämpfen. Der Rechtsstaat müsse zum Sozialstaat umgebildet werden ${ }^{27}$; aber nicht um dem Einzelnen ein soziales Netz zu bieten, das ihn notfalls auffängt, wenn er aus dem Wirtschaftskreislauf herausfällt. Vielmehr soll, wie Fichte es sich im Geschlossenen Handelsstaat dachte, die Wirtschaft von Regierungsseite aus so organisiert werden, daß sie alle Arbeitsfähigen, die zur Nation \{| S. 265\} gehören, an der Arbeit beteiligt und daß die Arbeit nicht eudämonistischen oder utilitaristischen Zwecken dient, sondern der nationalen Kultur, in deren Dienst sich auch die Wissenschaft zu stellen hat. ${ }^{28}$ Mit Zustimmung nimmt Rickert sogar Fichtes These auf, daß jede Nation als Kollektiv-Individuum eine spezifische Aufgabe habe, daß aber nur in der Kultur der Deutschen — als einem Urvolk — das Wesen der Menschheit zum Ausdruck gebracht werden könne. ${ }^{29}$

3. Nun haben wir die Elemente beisammen, die als Gründe in eine Erklärung der eingangs dargelegten Äußerungen Rickerts eingehen: Rickert sagt, was er sagt, weil er aus Gründen seiner philosophischen Überzeugungen genötigt ist, gewisse Momente an der nationalsozialistischen Bewegung gutzuheißen. Man kann nun auch sehen, welche Momente dies vor allem sind:

1. ein radikaler Nationalismus, der anti-kosmopolitische Implikationen hat und eine vage religiöse „Weihe“ erfährt (für die der Germanenkult der Nazis bei Rickert allerdings keine Rolle spielt);

2. die Verpflichtung der Politik auf einen nationalen Kulturstaat, d. h. auf die Pflege von Werten und die Verwirklichung von Zielen einer nicht nur politischen, sondern auch geistigen, aber nationalen Kultur;

3. eine allen anderen Nationen überlegene Legitimation, die der deutschen Kulturnation zugesprochen wird;

4. die Einschätzung, daß die Kulturideale der deutschen Nation spätestens seit dem Ausgang des Ersten Weltkriegs bedroht sind und sich nur bewahren lassen, wenn auch die Wissenschaften, insbesondere aber die Philosophie dafür in Dienst genommen werden.

Für den Fall einer Nazi-Herrschaft ist darin die Forderung enthalten, sich mit Ansichten über den Sinn des Lebens der durch diese Herrschaft geschaffenen Situation anzupassen, falls der Forderung in Rickerts Sicht nicht gewichtige Gründe entgegenstehen. Wie es sich damit verhält, ist noch zu erwägen. Aber schon jetzt kann man sagen: Wenn Rickerts Denken keine solchen Gründe enthält, erfolgt die Anpassung an den Nationalsozialismus nicht widerstrebend oder aus furchtsamem, vorlaufendem Gehorsam, sondern entspricht, wie Rickert selbst

\footnotetext{
${ }^{26}$ Rickert, Kant als Philosoph der modernen Kultur. Ein geschichtsphilosophischer Versuch, Tübingen 1924, 113.

${ }^{27}$ So lautet Rickerts Programmformel, die in den Vorlesungen der dreißiger Jahre mehrfach wiederholt wird. Der Hauptgrund für die Forderung, die sie formuliert, scheint jedoch nicht so sehr das Unrecht zu sein, das dem Einzelnen durch eine liberalistische Wirtschaftspolitik widerfährt, als vielmehr die Feststellung, daß der ethische Individualismus der Autonomie und das geschichts-individualistische Kulturideal der Nation bedroht sind, wenn wie im wirtschaftlichen Liberalismus und in der Ideologie der Sozialisten - das Streben nach Lust und Nutzen oberste Priorität bekommt. (Vgl. die Vorlesung über Sozialphilosophie vom Sommersemester 1932, Teil VI: Politik, ca. 32).

28 Ähnlich Heidegger in seiner Rektoratsrede.

${ }^{29}$ Fichte-Vorlesung, WS 1933/34, Teil X, 170 ff.
} 
behauptet, einer eigenen, in seiner Wertphilosophie verankerten Überzeugung. Einige weitere Motive, die Rickert mit den Nationalsozialisten verbinden, geben der Anpassungsforderung sogar zusätzlich Kraft:

5. Wie die Nationalsozialisten, wenn auch wohl aus anderen Gründen als sie, tritt Rickert dafür ein, den Rechtsstaat in einen Sozialstaat umzubauen, der das Recht auf Arbeit garantiert, aber die Arbeit staatlich organisiert, die Wirtschaft vom Weltmarkt abkoppelt und sie auf Autarkie umstellt;

6. Rickert hat den Ersten Weltkrieg nie als europäische Katastrophe, sondern immer nur als nationale Herausforderung verstanden; und den Versailler Frieden nicht als selbstverschuldetes Unglück, sondern als schmachvolles Unrecht. Mit vielen anderen glaubte $\{\mid$ S. 266 $\}$ er, in der Frage der Kriegsschuld schon gleich nach Kriegsende Bescheid zu wissen - bevor die Archive geöffnet wurden; und natürlich lag nach diesem Glauben die Schuld in der Hauptsache nicht bei den Deutschen. ${ }^{30}$

7. Gutheißen muß es Rickert auch, wenn im nationalen Interesse internationale Konventionen wie diejenige der Mitarbeit Deutschlands im Völkerbund aufgekündigt werden.

Auch mit den (als Nr. 5.-7.) genannten, zusätzlichen Motiven zu einer eventuellen Anpassung war Rickert nicht eo ipso ein Sympathisant oder gar Parteigänger der Nazis. Ebenso wie die zuvor angeführten Momente bestanden diese Motive ja auch schon in den Jahren, in denen Rickert Hitler noch ablehnte. Man kann es sich ersparen, über die Gründe der Ablehnung zu spekulieren. ${ }^{31}$ Denn im Jahr 1933 sind diese Gründe jedenfalls „durch Hitlers Erfolge“ entfallen oder nebensächlich geworden.

Das hat vermutlich mit drei weiteren Gesichtspunkten zu tun, die erst jetzt für Rickert motivierend werden:

8. In einer geschichtsphilosophischen Diagnose der Moderne hatte Rickert schon 1924 die Auffassung vertreten, daß eine Synthese im Kulturleben der Neuzeit nur noch durch eine Autorität möglich ist, die dem Streben nach Autonomie einzelner Kulturbereiche keine Erfüllung gewährleistet. ${ }^{32}$ Nun, angesichts der vermeintlichen Gefahr einer Katastrophe, von der ihm die deutsche Kultur vor allem durch ihre Zersplitterung in Extreme bedroht scheint, hält er wohl die Unterwerfung unter eine das Kulturleben diktatorisch reglementierende politische Autorität für die einzige Möglichkeit, die Gefahr abzuwenden; oder er hält diese Unterwerfung zumindest für das kleinere Übel im Verhältnis zur Fortdauer der Zersplitterung. $\{\mid$ S. 267\}

30 Vgl. den von Rickert unterzeichneten Hochschullehrerprotest gegen „die einseitigen KriegsschuldVeröffentlichungen“ im Heidelberger Tageblatt vom 8.1.1919. Darin wird behauptet, es gebe für die Deutschen (,gegenüber einer Vergewaltigung, die sich als vergeltende Gerechtigkeit aufspielen möchte“) „keine dringendere Pflicht, als die Kriegsschuld und den Eroberungswillen der Feinde immer von neuem vor der ganzen Menschheit festzustellen“. Andererseits aber macht der Protest darauf aufmerksam, daß die Archive noch geschlossen sind, aus denen sich der jeweilige Anteil an Kriegsschuld der einzelnen Staaten allenfalls ermitteln ließe; er fordert, „daß dem planlosen Treiben Einhalt getan werde, einseitig Dokumente der Öffentlichkeit preiszugeben, welche möglicherweise die deutsche Politik in selbstmörderischer Weise belasten könnten"; den Feinden aber wird unterstellt, daß sie „ihrerseits ihre internen Aktenstücke aus der Zeit vor dem Kriegsausbruch niemals der Welt offenbaren werden“. Wie dieser von insgesamt 75 Heidelberger Hochschullehrern unterzeichnete Protest seine Feststellungen und Forderungen miteinander verträglich halten soll, bleibt das Geheimnis seiner Verfasser. Trotzdem beteiligt sich Rickert an ihm. Insgesamt hat er zwischen 1917 und 1930 nur viermal öffentliche Stellungnahmen (durchweg „überparteilicher“ Intention) unterzeichnet (Vgl. Jansen, Auf dem Mittelweg nach rechts, a. a. O.) Umso signifikanter ist diese Beteiligung für seine politische Einstellung.

${ }^{31}$ Wenn man die Gesichtspunkte, die noch zu berücksichtigen sein werden, zu den schon registrierten hinzunimmt, darf man vermuten, daß die Ablehnung vor allem den plebeischen Zügen Hitlers und seiner Partei galt. Am Ende galt sie vielleicht nur noch ihnen.

${ }^{32}$ Rickert, Kant als Philosoph der modernen Kultur, a. a. O., 109. 
9. Der Beginn der Diktatur markiert für Rickert, wie wir sahen, einen epochalen Einschnitt. Mit ihm treten die politischen Fragen in den Vordergrund. Der nationale Staat legt fest, was Forderung des Tages ist, und diktiert die Bedingungen, denen man sich mit seinen Ansichten über den „Sinn des gegenwärtigen Lebens“ anpassen muß.

10. Gleichzeitig mit dieser Situationseinschätzung läßt sich Rickert auch ein Stück weit von der Aufbruchstimmung mitreißen, welche die Nazis für ihren politischen Umbau mobilisieren. Durch seine Faust-Interpretation hat er sich darauf sogar vorbereitet. Man vergleiche die nationalsozialistischen „Arbeitseinsätze“ mit dem entfesselten Aktionismus, den der erblindete Faust für den eigenen hält!

In summa dürfte Rickert die angeführten 10 Punkte als einen starken Grund für sein Verhalten im Jahr 1933 betrachtet haben. Er wollte mit diesem Verhalten „der historischen Situation“, wie er sie verstand, Rechnung tragen, indem er sich — nicht opportunistisch, sondern seiner philosophischen Überzeugung entsprechend — an die „Forderung des Tages“ anpaßte.

Trotzdem: Sollte der Wunsch, im „Kampf ums Dabeisein“ nicht zu kurz zu kommen, wirklich gar keine Rolle gespielt haben? Wird nicht auch ein Stück persönlicher Opportunismus für Rickert motivierend gewesen sein $?^{33}$ Eine sachgerechte, gut begründete Antwort hierauf ist kaum zu finden. Denn auch ein Handeln aus Sorge ums Schicksal der eigenen Philosophie hätte, wenn es den philosophischen Überzeugungen entsprach, keinen Opportunismusvorwurf verdient. Opportunistisch verhält sich ja nur, wer bei günstigen Gelegenheiten, den persönlichen Vorteil zu fördern, diesem Vorteil zuliebe gegen seine Überzeugungen handelt oder sie sogar aufgibt. Wie also müßten heute noch belegbare Begebenheiten ausgesehen haben, in denen das Schicksal der Philosophie nicht im Spiel war, Rickert aber sich unverkennbar zum eigenen Vorteil nazifreundlich verhielt, obwohl ihm seine philosophische Überzeugung etwas anderes nahegelegt hätte? Wer wüßte das zu sagen! Daran mag man erkennen, wie schwer aus einigem Zeitabstand gerechte moralische Urteile zu fällen sind.

Wichtiger als die Suche nach einer Basis für solche Urteile scheint mir die Frage: Warum hat sich Rickert von anderen Ingredienzen der nationalsozialistischen Weltanschauung und Politik — solchen nämlich, die seinen eigenen Überzeugungen zuwiderliefen — nicht so weit als möglich distanziert? Die Antwort hierauf ist verhältnismäßig leicht zu geben. Verfassungsgarantien dafür, daß bei der Bildung eines Sozialstaats wie des charakterisierten nicht die Substanz des Rechtsstaats verlorengeht, sind in der Rickertschen Wertphilosophie und Weltanschauung minimal. Jedenfalls sollen sie nicht in der Unverzichtbarkeit parlamentarischdemokratischer Strukturen bestehen und auch nicht in einer Präferenz für die Erhaltung eines Katalogs von Menschenrechten, sondern in den zwei schmächtigen Klauseln, daß der Staat jedem Bürger eine häusliche Sphäre möglichst unbeschränkter Betätigung seiner Individualität zu sichern habe ${ }^{34}$ und daß jedes Glied einer Nation zu einer autonomen Persönlichkeit werden soll. Beide Klauseln formulieren Forderungen. deren Erfüllung nach Unterordnung der universalistischen Werte unter den Wert der \{|S. 268\} Nation nur noch wenig Schutz gewähren kann — jedenfalls gegen den Verlust der Zugehörigkeit zur Nation und gegen kriminalisierende, Menschenrechte verletzende Rechtsetzung und -durchsetzung.

Ein Weiteres kommt hinzu: Die Zumutung, sich mit der eigenen außerwissenschaftlichen Weltanschauung dem anzupassen, was „Forderung des Tages“ ist, und das gleichzeitige Fehlen von Gründen, universalistische, inhaltliche Prinzipien des parlamentarisch-demokratischen Rechtsstaats zu verteidigen, wirken

\footnotetext{
${ }^{33}$ Das hat mir Eike Wolgast (Heidelberg) zu bedenken gegeben.

${ }^{34}$ Vorlesungs-Skript über Politik, abgeschlossen am 29.7.1932, 91 Seiten, 89.
} 
in fataler Weise zusammen. Sie berauben Rickert der Möglichkeit, sich gegen nationalsozialistisch motivierte Rechtsverletzungen zur Wehr zu setzen. Rickert wird nicht erst durch die Umstände hilflos, sondern zuvor durch eigene Grundsätze, die von ihm Anpassung fordern - auch da schon, wo die Machtverhältnisse noch nicht dazu zwingen. Zum Beispiel schreitet der Institutsdirektor Rickert nicht gegen einen Anschlag von Thesen ein, den nationalsozialistische Studenten am Philosophischen Seminar angebracht haben und durch den jüdische Kommilitonen diffamiert werden. Persönlich von einem jüdischen wissenschaftlichen Mitarbeiter am Seminar zur Rede gestellt weiß Rickert nur mit der Frage zu antworten, was sein Einschreiten denn „,genützt“ hätte. ${ }^{35}$ In derselben Weise dürfte sich erklären, warum Rickert seine Ablehnung sozial-darwinistischer und rassistischer Tendenzen im Weltanschauungsstreit seit dem Frühjahr 1933 nicht mehr zu erkennen gibt. So weit ging offenbar seine Anpassungsbereitschaft - ob sie nun opportunistisch war oder philosophisch, wenn auch schlecht, begründet.

\section{Ein kurzes Fazit für uns ${ }^{36}$}

Ich habe den Fall, der Heinrich Rickert mit partieller Identifikation dem Nationalsozialismus „zufallen“ ließ, bisher aus der Nahperspektive derjenigen Gegenwart zu betrachten versucht, in welcher er stattfand, — also nach Kräften davon abgesehen, daß wir den Ausgang der schlimmen Geschichte kennen, in die Heinrich Rickert und seine Philosophie hineingezogen wurde. Nun aber, nachdem der Fall dargestellt und erklärt ist, sollten wir von der Gegenwart des Jahres 1933 Abstand nehmen und uns fragen, was aus dem Erklärten gelernt werden kann. Das nämlich sind wir, glaube ich, den Opfern nationalsozialistischer Verbrechen vor allem schuldig — im Gedenken an sie und im Eingeständnis, daß Verbrechen sich durch üble Gedanken vorbereiten. Hierzu wenigstens noch einige Bemerkungen:

1. Rickerts Anpassung zeigt uns, wie leicht aus einem braven Demokraten und politisch eher Liberalen als Nationalkonservativen in einer schweren Krise der Weimarer Republik ein Anhänger Hitlers, des Diktators, werden konnte und wie wenig es bedurfte, vor dessen Untaten vorübergehend die Augen zu schließen. Eine Einstellung wie diejenige der bloßen „Vernunftrepublikaner“ oder gar ein antidemokratisches Denken waren für diese Konversion nicht erforderlich.

2. Die verhängnisvollen gedanklichen Voraussetzungen für solche Anfälligkeit waren nicht erst in der Staats- und Wirtschaftskrise zu Anfang der dreißiger Jahre erfüllt, sondern bereits viel früher: schon mit kulturphilosophischen Einstellungen und Ideen, die im Wilhelminismus und in der Gründerzeit entstanden waren, die zum Ersten Weltkrieg beige- $\{\mid$ S. 269\} tragen hatten und in ihm sowie durch seinen Ausgang bei den Gebildeten in Deutschland befestigt worden waren.

3. Es gab Barrieren, auch im Denken, die vor der Anfälligkeit schützen konnten. Aber einen wirklichen Schutz boten ziemlich wenige:

- ein klares Bewußtsein von der Unerläßlichkeit detaillierter, wirksam verteidigter Menschenrechte, von den Prinzipien des Rechtsstaats und von ihrer Unverzichtbarkeit auch in politischen sowie ökonomischen Notlagen;

\footnotetext{
${ }^{35}$ Vgl. den Beitrag von R. Klibanskv, in Unispiegel: 6/88 S. 3

${ }^{36}$ Ergänzendes hierzu enthält meine Abhandlung Krise und Untergang des süddeutschen Neukantianismus in ihren dritten Abschnitt. (In: H. J. Sandkühler, Hrsg., Philosophie im Nationalsozialismus. Hamburg 2009. S. 83-132, darin insbes. S. 97-123)
}

in neu durchgesehener und ergänzter Autorenfassung (März 2014) 
- Einsicht in die überlegene politische Vernünftigkeit der Verfassung einer parlamentarischen Demokratie insbesondere bei den Politikern, d. h. ihre Verpflichtung auf formale parlamentarische Verfahrensrationalität;

- eine hochentwickelte Sensibilität für die Gefahren des Antisemitismus und verwandter sozialpsychologischer Diskriminierungsmechanismen. Bloße Aversion gegen den Antisemitismus oder gute Beziehungen zu jüdischen Freunden schützten in der Regel nicht davor, diesen Gefahren zu erliegen.

4. Ein verhängnisvolles Unternehmen, die Barrieren abzubauen — womöglich in der Meinung, die ethische Autonomie der Bürger zu stärken —, ist die Tendenz gewesen, rechtsstaatliche Imperative durch sozialstaatliche Forderungen zu überbieten und im Not- oder Konfliktfall den sozialstaatlichen Gütern vor den rechtsstaatlichen Vorrang zu geben. Das sollte uns sehr vorsichtig machen gegenüber dem Verlangen, ein Recht auf Arbeit in unserer Verfassung zu verankern, bevor der Staat durch verläßliche Mittel seiner Wirtschaftspolitik garantieren kann, daß Ansprüche, die sich aus einem solchen Recht ergeben, unbeschadet anderer Rechte erfüllt werden.

5. Eine Wertphilosophie, welche wie diejenige Rickerts alle denkbaren Werte in acht Klassen sortiert und anhand weniger Beispiele definitiv unter den Gütern, an denen Werte solcher Klassen „haften“, Präferenzen ausmachen will, ist ein schlechter Ratgeber, wenn es um prinzipielle Fragen des Rechts, der Gerechtigkeit und der Staatsgesundheit geht. Eine Devise gar, in diesen Fragen dem Nationalismus zuliebe Kant durch Fichte zu „vollenden“, ist von Übel. Im Rückblick auf Rickerts Bildungsgeschichte muß man sogar sagen: sie war die Wurzel des Übels. 